\title{
Parasitoides púpales de moscas (Diptera) asociadas al estiércol de ganado bovino, en Aragua, Venezuela
}

\section{Pupal parasitoids of flies (Diptera) associated to the bovine cattle manure, in Aragua, Venezuela}

\author{
Montilla-Coronado Rafael Paúl*
}

\begin{tabular}{l}
\hline Datos del Artículo \\
\hline Laboratorio de Entomología de la Unidad \\
de Protección Vegetal (UPV). \\
Centro Nacional de Investigaciones Agro \\
pecuarias (CENIAP). \\
Instituto Nacional de Investigaciones Agrí \\
colas (INIA). \\
Av. Universidad Vía El Limón, Apdo. \\
588, Maracay 2101, \\
Edo. Aragua. Venezuela. \\
(0243) 2404978, 2404827-870. A \\
*Dirección de contacto: \\
Rafael Paul Montilla-Coronado \\
E-mail: rmontilla@inia.gob.ve \\
rmoncor7@gmail.com. \\
\hline
\end{tabular}

\section{Palabras clave:}

Parasitoidismo,

control biológico,

sistemas de producción de leche,

moscas plagas,

salud animal,

entomófago pupal,

taxonomía avispas,

Hymenoptera.

\section{J Selva Andina Biosph.} 2017; 5(2):124-132.

\section{Historial del artículo.}

Recibido abril, 2017.

Devuelto agosto 2017

Aceptado septiembre, 2017

Disponible en línea, noviembre 2017.

Editado por:

Selva Andina

Research Society

\section{$\underline{\text { Key words: }}$}

Parasitoidism,

biological control,

milk production systems,

pests flies,

animal health,

pupal entomophagus,

wasp taxonomy,

Hymenoptera.

\section{Resumen}

En explotaciones pecuarias, las moscas constituyen uno de los principales problemas sanitarios, por las enfermedades que transmiten y el stress que ocasionan sobre los animales, con las consecuentes pérdidas económicas. Con el fin de obtener información sobre las especies de moscas presentes y los parasitoides pupales asociados, en un sistema de producción de leche de ganado bovino, de una localidad del estado Aragua, se colectaron pupas de moscas en los estiércoles encontrados en los potreros, y en las pilas que se hacen cuando limpian las salas de ordeño, las mismas fueron puestas en cría en el insectario del Instituto de Zoología Agrícola de FAGRO-UCV, en Maracay, estado Aragua. Los adultos emergidos fueron preservados en viales con alcohol al 70\% hasta su montaje e identificación. Las moscas que emergieron de los potreros, fueron determinados como Themira sp., (Sepsidae), Fannia sp., (Muscidae), y Musca sp., (Muscidae), de las pilas de estiércol como Musca domestica (Muscidae), Leptocera sp., (Sphaeroceridae) y Physiphora sp., (Ulidiidae). Entre los parasitoides que emergieron de las pupas de moscas se identificaron a Spalangia imitator y Spalangia cameroni (Pteromalidae), Trichopria sp., (Diapriidae) y Kleidotoma sp., (Figitidae). El grado de parasitoidismo fue bastante bajo $(<5 \%)$. Todos estos registros son nuevos aportes de entomófagos asociados a sistemas de producción pecuario en el país, que con investigación adecuada, pueden ser aprovechados para la elaboración de estrategias de control de moscas problemáticas y por las industrias de masificación de controladores biológicas de tales plagas.

(C) 2017. Journal of the Selva Andina Biosph. Bolivia. Todos los derechos reservados.

\section{Abstract}

In livestock farms, flies are one of the main health problems, due to the diseases they transmit and the stress they cause on animals, with the consequent economic losses. In order to obtain information on the species of flies present and associated pupal parasitoids in a system of milk production of cattle from a locality of Aragua state, pupae of flies were collected in the manures found in the paddocks, and In the piles that are made when cleaning the milking rooms, The same ones were put in breeding in the insect of the Institute of Agricultural Zoology of FAGRO-UCV, in Maracay, Aragua state. The emerged adults were preserved in vials with $70 \%$ alcohol until their assembly and identification. The flies that emerged from the pastures were determined as Themira sp., (Sepsidae), Fannia sp., (Muscidae), and Musca sp., (Muscidae), manure stacks such as Musca domestica (Muscidae), Leptocera sp., (Sphaeroceridae) and Physiphora sp., (Ulidiidae). Among the parasitoids that emerged from the pupae of flies were Spalangia imitator and Spalangia cameroni (Pteromalidae), Trichopria sp., (Diapriidae) and Kleidotoma sp., (Figitidae). The degree of parasitoidism was quite low $(<5 \%)$. All these records are new contributions to livestock production systems in the country, that with proper research, which can be used for the development of strategies for control of problem flies and for the industries of massification of biological controllers of such pests. 


\section{Introducción}

Las moscas son consideradas muy importantes por su gran diversidad de especies, muchas de las cuales poseen distribución cosmopolita, estando presente en lugares que poseen climas desde subpolares hasta tropicales. Su importancia es mayor en áreas urbanas y suburbanas, asimismo en explotaciones pecuarias y avícolas, donde influyen directamente o indirectamente en el incremento de los costos de producción, debido a la capacidad que tienen de transportar agentes patógenos, responsables de diferentes enfermedades en animales. Adicionalmente las moscas provocan problemas al invadir las áreas residenciales, vecinas a los sitios de reproducción y cría de animales con diferente propósito comercial (Vergara-Ruiz 1996, Cadena et al. 1997, Hogsette et al. 2001, Vergara-Ruiz \& Jiménez 2015).

Investigaciones han determinado que las moscas, en especial la mosca doméstica, puede transmitir a humanos y animales enfermedades causadas por protozoos, bacterias, virus, y lombrices parásitas (Quiceno et al. 2010). Los dípteros son transmisores de más de 65 enfermedades en humanos que pueden ser mortales, entre las cuales se señalan: fiebre tifoidea, anaplasmosis, disentería, cólera, poliomielitis, lepra, tuberculosis, además algunas producen miasis con cuadros clínicos variables en humanos y animales (Carles-Tolrá 1997, Calderón-Romero 2004).

En la explotaciones pecuarias se ha encontrado que las especies de moscas que mayormente se encuentran en los rebaños bovinos y en menor porcentaje en explotaciones avícolas son: Musca domestica (L), Haematobia irritans (L), Dermatobia hominis (L), Cochliomyia hominivorax (Coquerel), Musca autumnalis DeGeer (Blume 1986). En inventarios de poblaciones de moscas en instalaciones ganade 125 ras y avícolas se ha determinado que las dos primeras especies citadas representan el $90 \%$ de la población (Vergara \& Jiménez 2015).

Algunas moscas provocan irritación continua al alimentarse de las secreciones de los ojos, nariz y heridas, que afectan el proceso de alimentación de los animales en cría, ocasionando reducción significativa en la producción de carne, leche, huevos y por consiguiente pérdidas económicas al empresario o productor agrícola (Stork 1979).

Actualmente las moscas están siendo controladas por diversos enemigos naturales, entre los cuales destacan algunos hongos entomopatógenos y varias especies de parasitoides, que controlan alrededor del $50 \%$ de las pupas de dichos insectos (Godfray 1994, Cova et al. 2010), entre avispas parasitoides de pupas de moscas se mencionan Muscidifurax sp. (Girault and Sanders 1910), Spalangia sp. (Latreille 1805), Pachycrepoideus sp. Ashmead (Blume 1986, Hogsette et al. 1994, Schlapbach 2017, Nicholls 2008, Gibson 2009, Marchiori et al. 2010).

La aplicación del control biológico constituye una herramienta efectiva y significativamente más económica y ecológica que otros métodos de control (Treviño 2007), en Venezuela el control de moscas es netamente químico.

Para los años 60, se introdujo en Costa Rica al parasitoide pupal Pachycrepoideus vindemmiae Rondani (Hymenoptera: Pteromalidae), que según Carballo (2002) fue muy eficiente en el control de dípteras en ambientes naturales y en granjas, con parasitoidismos de $69.5 \%$ promedio. En México en explotaciones bovinas para la producción de leche, fueron identificados Muscidifurax sp., y Spalangia spp., ejerciendo buen control sobre poblaciones de las moscas Stomoxys calcitrans (L) (Diptera: Musci- 
dae) y M. domestica (Nava-Camberos et al. 2000, Hernández-Hernández et al. 2004, Ávila-Rodríguez et al. 2015). Spalangia drosophilae (Ashmead) fue encontrado parasitando pupas de Archisepsis scabra Loew (Diptera: Sepsidae) en heces de bovinos lecheros en Brasil (Marchiori 2001). Aguiar-Menezes et al. (2003), registran la presencia de los parasitoides pupales $P$. vindemmiae, Spalangia endius Walker y Coptera haywardi Loiácono (Hymenoptera: Diapriidae) parasitando pupas de Tephritidae. Sivinski et al. (1998), estudiaron y compararon el potencial biológico de los parasitoides de pupas de moscas C. haywardi, Dirhinus himalayanus Westwood (Hymenoptera: Chalcididae) y Spalangia geminae Boucek (Hymenoptera: Pteromalidae). Vergara-Ruiz \& Jiménez (2015) señalan que países como Argentina, Colombia y Estados Unidos de América, han obtenido importantes logros con la liberación de Spalangia cameroni Perkins (Hymenoptera: Pteromalidae) para el control de pupas de moscas.

En Venezuela son pocos los trabajos que se han realizado sobre parasitoides de moscas asociadas al estiércol de ganado. Peraza (1990), realizó un trabajo sobre la biología de $S$. cameroni parasitoide de M. domestica, señalando que es un factor biótico que limita la densidad poblacional de la plaga. Entre 1987 y 1992 fueron introducidas tres especies de Pteromalidae (S. cameroni, S. endius, M. raptor) en la Región Centroccidental de Venezuela (Ferrer 2001), sin hasta ahora disponer de información de su efecto de control sobre moscas de importancia agrícola como plagas.

Aunque el uso del control biológico de moscas en estos sistemas de producción no está generalizado (Dean 1982), es importante emprender acciones que garanticen, potencien y preserven poblaciones de depredadores y parasitoides naturales (De Bach 1968, Rutz \& Patterson 1990), que contribuyan a mantener poblaciones de plagas por debajo del nivel de daño económico. Es de destacar que aún no se conoce bien los aspectos fisiológicos, ecobiológicos y poblacionales tanto de las plagas como de los controladores naturales en estos sistemas de producción agrícola, por lo que la investigación en este campo del manejo integrado de plagas debe ser abordado sin más retraso. En este sentido, este trabajo busca contribuir con el conocimiento de insectos entomófagos parasitoides de moscas asociadas a explotaciones bovinas lecheras del país.

\section{Materiales y métodos}

Sección de bovinos de FAGRO, Maracay edo. Aragua, $12^{\circ} 16^{\prime} \mathrm{N}-67^{\circ} 36^{\prime} \mathrm{W}$, mayo-octubre (lluvia) y Noviembre-Abril (sequía), precipitación acumulada $842.4 \mathrm{~mm}$ anual, temperatura promedio anual 30 ${ }^{\circ} \mathrm{C}$, evapotranspiración potencial 1900-2000 $\mathrm{mm} /$ anual, HR 65\%, Bosque seco tropical (Servicio Climatología FAGRO-UCV 1999, CENIAP 2009).

La estación de experimentación de bovinos del departamento de producción animal cuenta con un área de 12 ha, con 15 potreros de 0.8 ha cada uno, utilizados para pastoreo libre del ganado después que salen de la actividad de ordeño, todo el ganado es mestizo (5/8 Holstein-3/8 Brahman).

Los muestreos se realizaron en la época seca entre los meses Diciembre 2009 y marzo 2010, donde los valores de precipitación fueron 842.4-30.7 y temperatura 30.8-34.8 ${ }^{\circ} \mathrm{C}$. En cada fecha de muestreo, se seleccionaron cuatro potreros al azar y dentro de estos se marcaron dos áreas (llamadas pastoreo y descanso), es de resaltar que los animales al salir del pastoreo, dejan en el potrero sus bostas o deposiciones fecales (estiércol), en este sentido a los siete y quince días de dejadas las deposiciones, se seleccionaban ocho por cada área marcada, para un total de 
dieciséis por potrero. Dichas deposiciones eran llevadas al laboratorio de entomología del Museo del Instituto de Zoología Agrícola (MIZA), FAGROUCV, en Maracay, Edo. Aragua, envueltas en papel periódico y cargadas en bolsa plásticas debidamente identificadas para su revisión. Una vez en el laboratorio, las muestras fecales fueron sometidas a un proceso de desmenuzado suave y flotación en bañeras contenedoras de agua. Las pupas viables fueron recolectadas y separadas con cuidado del estiércol, y desinfectadas con una solución de hipoclorito al 5\%, durante cinco minutos, luego fueron colocadas individualmente en frascos de vidrio de $5 \mathrm{~mL}$, colocando en el fondo papel absorbente humedecido con agua destilada, y tapados con tapa de algodón para facilitar la aireación.

Por otro lado se recobraron pupas de moscas del estiércol apilado proveniente de la limpieza del área de levante, el cual era depositado a la intemperie en un área alejada de los corrales, para el muestreo se seleccionaron las pilas por el tiempo de apilado (cero (0), siete (7) y más de quince (15) días), en cada pila se procedió a extraer las pupas de moscas en tres cuadrantes de dimensiones $20 \times 20 \times 10 \mathrm{~cm}$, una vez por semana, durante tres semanas, que luego eran llevadas al laboratorio de entomología, si- guiendo la misma metodología mencionada para su cría.

Diariamente se revisaban los frascos, todo el material emergido era capturado y preservado en alcohol etílico al $75 \%$, hasta su montaje entomológico y posterior identificación. Para la identificación se utilizó una lupa binocular, marca Leica M8, con aumento máximo de 500X, también se empleó una lámpara de luz blanca fría, con difusores de luz hechos en papel pergamino, para la identificación de los parasitoides se emplearon las claves de Gibson et al. (1997), Masner \& García (2002), Fernández \& Sharkey (2006), Gibson (2009), y para las moscas hospedadoras, las claves de Borror et al. (1976) McAlpine et al. (1987), así como con la colaboración de Norma Díaz (Museo de La Plata, Argentina) para la identificación de los parasitoides Cynipoidea, y de José Luís García (MIZA) para los Proctotrupoidea. No se empleo ningún método estadístico para el trabajo.

La incubación de las pupas se realizó en condiciones ambientales naturales. Todo el material entomológico recobrado se encuentra depositado en el MIZA.

\section{Resultados}

Tabla 1 Valores promedios totales de moscas y parasitoides emergidos en los estiércoles por mes de colecta

\begin{tabular}{ccccc}
\hline Fecha Colecta & $\begin{array}{c}\text { Promedio pupas } \\
\text { colectadas }\end{array}$ & $\begin{array}{c}\text { Promedio moscas } \\
\text { Emergidas }\end{array}$ & $\begin{array}{c}\text { \% Moscas emer- } \\
\text { gidas }\end{array}$ & $\begin{array}{c}\text { Promedio parasi- } \\
\text { toides Emergidos }\end{array}$ \\
\hline Diciembre 2009 & 374.75 & 336.50 & 96.25 & 20.19 \\
Enero 2010 & 312.50 & 298.00 & 89.71 & 13.41 \\
Febrero 2010 & 623.25 & 561.85 & 87.46 & 28.09 \\
Marzo 2010 & 266.75 & 258.15 & 86.80 & 11.62 \\
\hline Totales & 1577.25 & 1454.50 & & 73.31 \\
\hline
\end{tabular}


Figura 1 Vista lateral Themira sp.

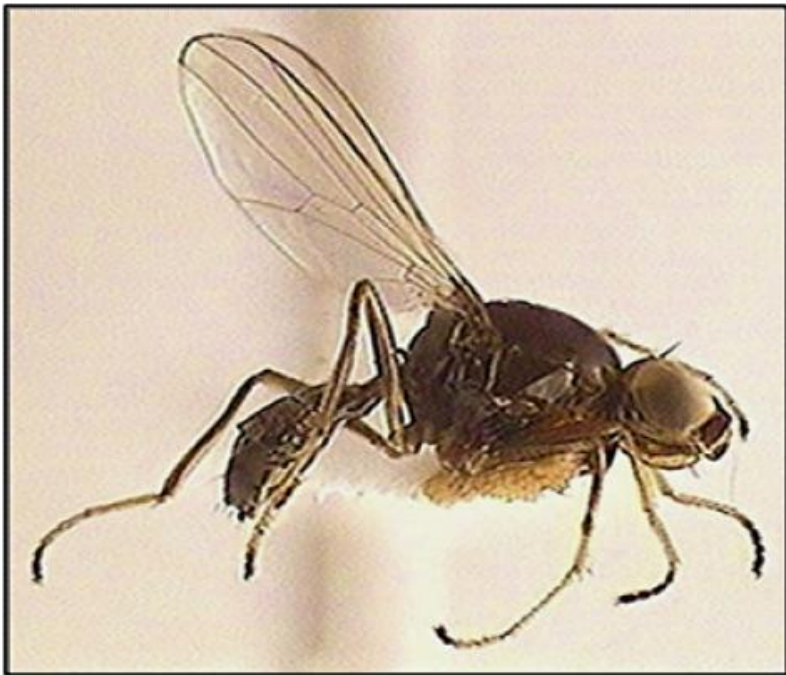

Figura 2 Vista dorsal Fannia sp.

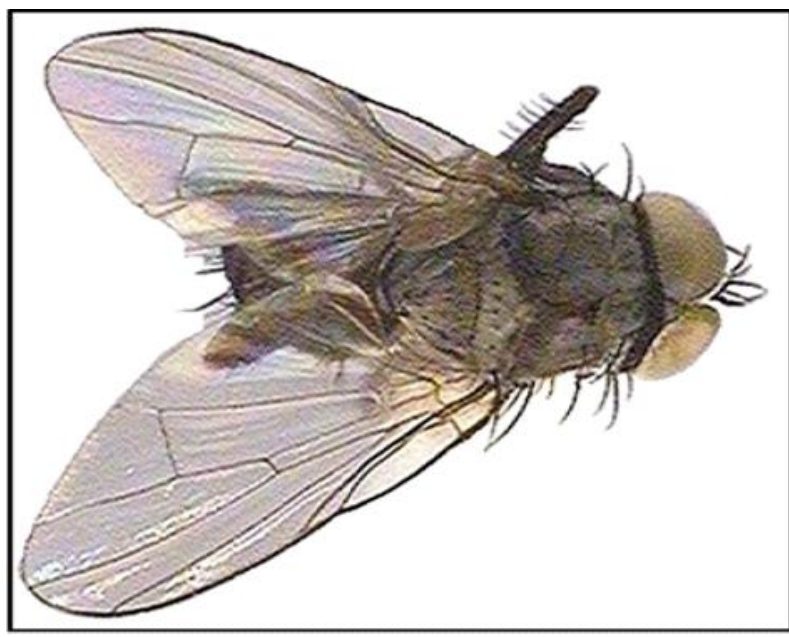

Figura 3 Vista dorsal Physiphora

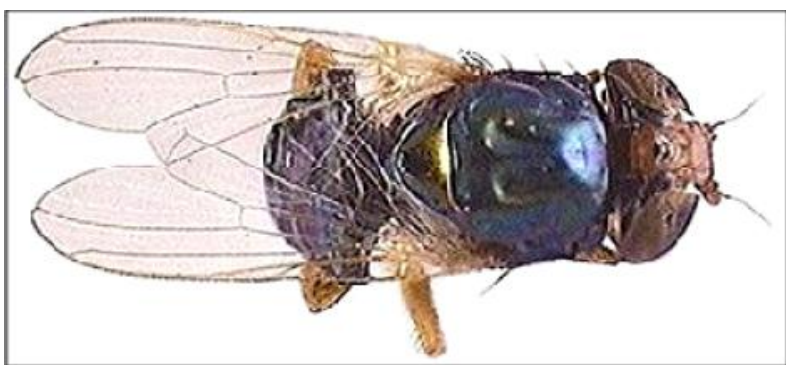

Figura 4 Vista lateral Spalangia cameroni

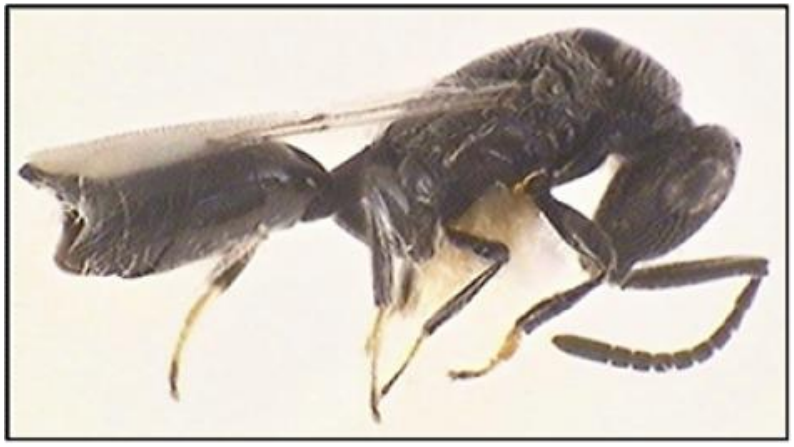

Figura 5 Vista lateral Trichopria sp.

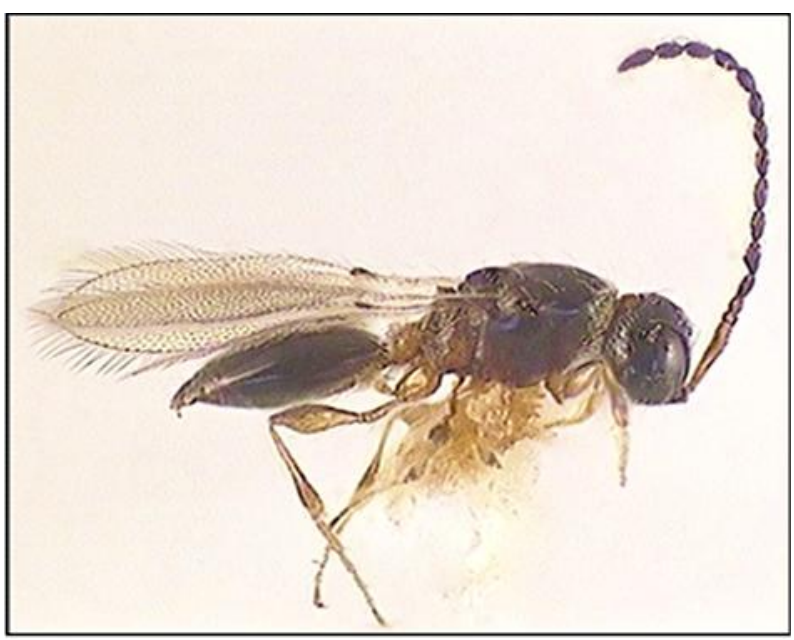

Figura 6 Vista lateral Kleidotoma sp.

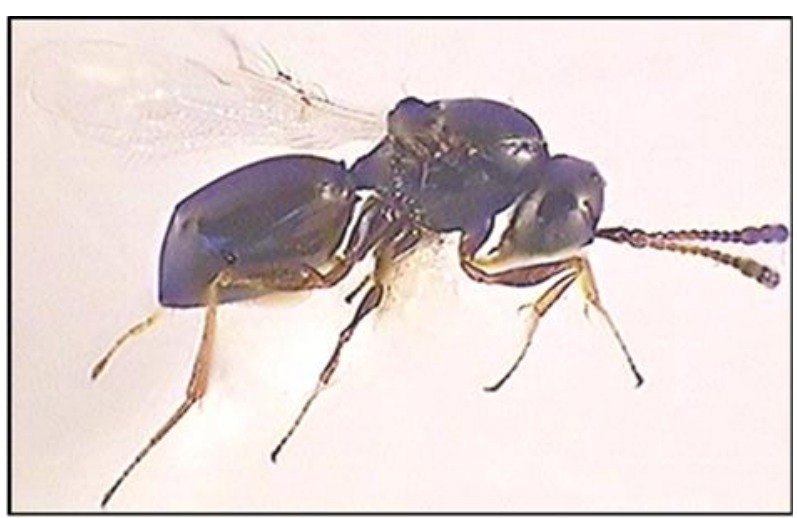




\section{Discusión}

En total se encontraron 5 especies de dípteros, 2 en los estiércoles colectados en los potreros y 3 de los provenientes de los depositados a la intemperie, en ambos casos las especies obtenidas no fueron las que se esperaban, pues salvo $M$. domestica que apareció en los potreros, las demás no son las comúnmente encontradas en estos medios, como $S$. calcitrans, H. irritans, D. hominis y C. hominivorax (Vergara-Ruiz \& Jiménez 2015).

Entre las especies de moscas (Diptera) encontrados se identificó a Themira sp. Robineau-Desvoidy (Sepsidae), Fannia sp. Robineau-Desvoidy (Muscidae), y M. domestica Linneo (Muscidae), que emergieron de los potreros, Leptocera sp., Olivier (Sphaeroceridae), Physiphora sp., Fallén (Ulidiidae), que emergieron de las pilas. Todos los parasitoides que emergieron de la cría de moscas pertenecen al orden Hymenoptera, y son endoparasitoides pupales, los mismos fueron identificados como Spalangia imitator Gibson y Spalangia cameroni Perkins (Pteromalidae), Trichopria sp., Ashmead (Diapriidae) y Kleidotoma sp., Westwood (Figitidae). Aunque no era el objetivo del trabajo se determinó que el grado de parasitoidismo en todos los casos, fue bastante bajo $(<5 \%$ ) (Tabla 1$)$, aspecto que se atribuye a las fechas en que se realizaron las tomas de muestras, que correspondieron plenamente a la época de sequía en nuestro país, donde la incidencia de moscas es baja.

Es de destacar que la captura de especies de Spalangia sp., Trichopria sp., y Kleidotoma sp., en un área experimental de potreros, así como la información de distribución reflejada en la revisión bibliográfica, hace suponer que estos géneros tienen un distribución geográfica más amplia y es posible que estén ejerciendo una cierta acción de control de 129 moscas vectores de enfermedades en explotaciones ganaderas, así como de sistemas agrícolas en general, lo cual no ha sido suficientemente evaluado, y que propone líneas de investigación para proyectos adelantados de manejo integrado de plagas en sistemas de explotación pecuaria.

La mayor cantidad de moscas se observa entre los primeros cinco días de la deposición del material fecal (estiércol), donde las condiciones de humedad del estiércol es máxima, ya que con el transcurrir del tiempo ocurre la deshidratación, que se ve favorecida por la falta de remoción y por las altas temperaturas internas de la pila $\left(64-75^{\circ} \mathrm{C}\right)$, lo cual limita el desarrollo de larvas de moscas, aunque también el de sus insectos entomófagos (Cadena et al. 1997, Orrego 2004).

Todos estos registros son nuevos aportes a sistemas de producción pecuarios en el país, que con mayor investigación pueden ser aprovechados para la elaboración de estrategias de control de moscas problemáticas y de bioindustrias de masificación de insectos entomófagos como controladores biológicas de tales plagas.

\section{Conflictos de intereses}

El autor declara que no existe ningún tipo de conflicto de interés en la planificación, realización y redacción del trabajo presente, con las instituciones nombradas, ni con los que apoyaron económicamente esta investigación.

\section{Agradecimientos}

Especial agradecimiento a la Dra. Norma Díaz, del Museo de la Universidad Nacional de la Plata, Tucumán, Argentina, por la identificación del Figitidae 
y al Dr. José Luís García Rodríguez del MIZA por la identificación del Diapriidae.

\section{Literatura citada}

Aguiar-Menezes EL, Menezes-Euripedes B, Loiácono-Marta S. First record of Coptera haywardi Loiácono (Hymenoptera: Diapriidae) as a parasitoid of fruit-infesting Tephritidae (Diptera) in Brazil. Neotrop Entomol 2003; 32(2): 355-8.

Ávila-Rodríguez V, Martínez-López R, Nava-Cam beros U, Sosa-Rubio M. Parasitoides de Pupas (Hymenoptera: Pteromalidae) de Moscas (Diptera: Muscidae) en Establos Lecheros de Jalisco, México. Southwest Entomol 2015;40(1): 141-50.

Blume RR. A checklist, distributional record, and annotated bibliography of the insects associated with bovine droppings on pastures in America north of Mexico. Southwest Entomol 1986; 9:1-55.

Borror DJ, DeLong DM, Triplehorn CA. $4^{\text {th }}$ Ed. An introduction to the study of insects. Holt, Rinehart and Winston. New York, Chicago. ISBN 0-03-088406-3; 1976. p. 768.

Cadena WD, Peláez JD, Vergara R. Evaluación del manejo integrado de moscas comunes en explotaciones pecuarias en Antioquia. Rev Colomb Entomol 1997;23(3-4):143-55.

Calderón-Romero L, Tay-Zavala J, Sánchez-Vega JT, Ruíz-Sánchez D. Los artrópodos y su importancia en medicina humana. Rev Fac Med UNAM 2004;47;(5):192-9.

Carballo M. Manejo de insectos mediante parasitoides. En: Avances en el fomento de productos fitosanitarios no sintéticos. Manejo integrado de plagas y agroecología. Costa Rica. 2002;66: 118-22.

Carles-Tolrá M. Los dípteros y el hombre. Boletín de la Sociedad Española Aragonesa, 1997;20: 405-25.

Centro Nacional de Investigaciones Agropecuarias (CENIAP). Portal de red de climatología. 2009 [Citado 2016 julio 06]. Disponible en: http:// www.ceniap.gob.ve.

Cova LJ, Vicente-Scorza DJ, García DE, Cañizález LM, Guedez CC, Maffey M, et al. Control temporal de moscas (Musca domestica) en galpones avícolas mediante nebulizaciones con conidias de Beauveria brongniartii. Zootec Trop 2010;28(1):9-15.

De Bach P. Control biológico de los insectos plagas y malas hierbas. Edición revolucionaria. Instituto del Libro. La Habana, Cuba; 1968. p. 949.

Dean GJ. Phenology of aphidophagous predators. Ann Appl Biol 1982;101:182-4.

Fernández F, Sharkey MJ, editores. Introducción a los Hymenoptera de la Región Neotropical. Sociedad Colombiana de Entomología y Universidad Nacional de Colombia, Bogotá D.C; 2006. p. 894.

Ferrer F. Biological control of agricultural insect pests in Venezuela: advances, achievements, and future perspectives. Biocontrol 2001;22: 67-74.

Gibson GAP, Huber JT, Woolley JB, editors. Annoted keys to the genera of Nearctic Chalcidoidea (Hymenoptera). NRC Research Press, Otawa, Ontario, Canada; 1997. p. 794.

Gibson GAP. Revision of New World Spalangiinae (Hymenoptera: Pteromalidae). Zootaxa 2009; 2259: 1-159.

Godfray HC. Parasitoids: behavioral and evolutionary ecology. Princeton University Press, Prince ton New Jersey; 1994. p. 473. 
Hernández-Hernández B, Cruz-Vázquez C, González-Hernández C, Perales-Segovia C, Martínez-Martínez L. Parasitoides de pupas (Hymenoptera: Pteromalidae) de moscas (Diptera: Muscidae) asociados al estiércol de ganado lechero en Aguascalientes, México. Folia Entomol Mex 2004;43(1): 9-15.

Hogsette J, Farkas R, Coller R. Hymenopteran pupal parasites recovered from house fly and stable fly (Diptera: Muscidae). Pupae collected on livestock and poultry facilities in Northern and Central Hungary. Environ Entomol 1994; 23(3):778-81.

Hogsette JA, Farkas R, Thurocxy C. Hymenoptera pupal parasitoids recovered from house fly and stable fly (Diptera: Muscidae) pupae collected on livestock facilities in southern and eastern Hungary. Environ Entomol 2001;30(1):107-11.

Marchiori CH, Barbaresco LF, Ferreira ME. Parasitoides de dípteros coletados em excrementos de animais no sul de Goiás, Brazil. Arq Bras Med Vet Zootec 2010;62(1):236-9.

Marchiori CH. Spalangia drosophilae (Ashmead) (Hymenoptera: Pteromalidae) como inimigo natural de Archisepsis scabra (Loew) (Diptera: Sepsidae) em fezes bovinas. Arq Bras Med Vet Zootec 2001;53(6):663-5.

Masner L, Garcia R JL. The genera of Diapriinae (Hymenoptera: Diapriidae) in the World. Bull Am Mus Nat Hist 2002;268:1-138.

McAlpine JF. 94 Curtonotidae. In: McAlpine, J.F., Peterson, BV, Shewell GE, Teskey HJ, Vokeroth JR, Wood DM. (Coordinated by/JF McAlpine (Ed.)/DM Wood) Manual of Nearctic Diptera 2. Biosystematics Research Centre (formerly Institute), Ottawa, Ontario, Research Branch, Agriculture Canada, Monograph No. 28; 1987. p. 1007-10.
Nava-Camberos U, Gómez-Gallegos MY, RamírezGómez M. Determinación de parasitoides como agentes de control biológico de moscas de establo en Bermejillo, Durango. Rev Chapingo Ser Zonas Áridas 2000; 85-91.

Nicholls C. Control biológico de insectos: un enfoque agroecológico. Medellín. Editorial Universidad de Antioquia. Colección ciencia y tecnología. ISBN 978-958-714-186-3; 2008. p. 282.

Orrego P. Curso de compost y sus condiciones de manejo. Centro de apoyo rural CEAR. 2004 [Citado 2016 diciembre 06]. Disponible en: http://www.cb.peru.cl/initihuasi.

Peraza M. Biología de Spalangia cameroni Perkins (Hymenoptera: Pteromalidae) parasitoide de la mosca domestica Musca domestica L. (Diptera: Muscidae). [Tesis de Licenciatura]. Universidad Centro Occidental Lisando Alvarado (UCLA). Venezuela; 1990.

Quiceno J, Bastidas X, Rojas D, Bayona M. La Mosca doméstica como portador de patógenos microbianos, en cinco cafeterías del norte de Bogotá. Rev Udcaactual Divulg Cient 2010; 13(1): 23-9.

Rutz DA, Patterson RS, editors. Biocontrol of Arthropods Affecting Livestock Poultry. Westview Press, Inc., Boulder, Colorado, USA; 1990. p. 7-10.

Schlapbach FA. Control integrado de moscas. Novartis Argentina. IN: Universo porcino. El portal del cerdo. 2017 p. 1-18. [Citado 2017 marzo 08]. Disponible en: http://www.aacporcinos .com.ar/articulos/control_de_moscas_en_porci nos_2.html.

Servicio Climatología Facultad de Agronomía (FAGRO), Universidad Central de Venezuela (UCV). Departamento de Ingeniería de FAGRO-UCV. Maracay, Edo. Aragua; 1999. 
Sivinski J, Vulinec K, Menezes E, Aluja M. The bionomics of Coptera haywardi (Ogloblin) (Hymenoptera: Diapriidae) and other pupal parasitoids of Tephritid fruit flies (Diptera). Biol Control 1998;11:193-202.

Stork B. Guía para el reconocimiento de moscas de importancia pecuaria. 1979 [Citado 2016 julio 06]. Disponible en:

http://www.inia.cl/intihusi/entomologia.

Treviño R. Insectos benéficos para la ganadería. México. 2007 [citado 2016 noviembre 28]. Disponible en: http://www.kunafin.com.
Vergara-Ruiz R, Jiménez J. Manejo integrado de moscas en explotaciones pecuarias. IN: XLII Congreso de la Sociedad Colombiana de Entomología SOCOLEN. Julio 29, 30 y 31 de 2015. pags. 231-254. 2015 (citado 2017 enero 16). Disponible en: http://www.socolen.org.co/ secretariacongres

Vergara-Ruiz R. Sistema de manejo integrado de moscas comunes en explotaciones pecuarias: alternativa ecológica y económica. IN: Epidemiología, diagnóstico y control de enfermedades parasitarias en bovinos. CORPOICA, Regional 4, Compendio 2, Medellín; Noviembre de 1996. p. 41-50. 